\title{
Evaluation of Inter-pair Differences in Caries among South Indian Monozygotic Twins: A Cross-sectional Study
}

\author{
Rita Zarina ${ }^{1}$, Sobha Kuriakose ${ }^{2}$, Jaini Lalithamma ${ }^{3}$, Anna D’Souza ${ }^{4}$, Bharathiammal Padmakumari ${ }^{5}$, Puthiyapurayil Jeeva ${ }^{6}$
}

\begin{abstract}
Introduction: Assessment of difference in caries prevalence among twins may help determine the character and the extent of the influence exercised by the environment, as a complement of the genetic constitution.

Aim: To investigate the inter-pair differences in caries prevalence among South Indian monozygotic twins in relation to birth weight, birth order, and gender. Possible correlation among other attributes such as breastfeeding, socioeconomic status, oral habits, frequency of brushing, and sweet intake were also assessed.

Materials and methods: A sample of 40 monozygotic children between the age group of 6 and 12 was selected. The parents were asked to complete the questionnaire and report with their children. Then dental examinations were carried out and the prevalence of dental caries among the twins were assessed.

Results: Among the total samples, $61.5 \%$ exhibited caries. Lower birth weight twins showed an increased chance of caries (72.22\%), compared to higher birth weight twins (68.18\%). Second of the twins had an increased risk of dental caries (70\%) compared to the first (60\%). $90 \%$ of the male twins and $45 \%$ of the female twins had caries. The association between dental caries and other variables were also assessed; those who were exclusively breastfed had an increased risk (70\%) of caries. Evaluation of socioeconomic status and caries revealed a risk of for the high $(65 \%)$, middle $(62.5 \%)$, and lower groups (58.33\%). Children without oral habits showed a higher prevalence $(73.3 \%)$ of caries when compared to those without any habits (50\%). It was noticed that the brushing frequency is not related to the severity of caries. The strongest association was noticed between excessive sweet liking and prevalence of dental caries.

Conclusion: The study revealed difference in caries prevalence among monozygotic twins in relation to birth weight, birth order, and gender. It was found that variations in caries prevalence existed with other attributes also.

Keywords: Breastfeeding, Caries prevalence, Cross-sectional study, Frequency of brushing, Monozygotic twins, Oral habits, Socioeconomic status, South Indian, Sweet intake. International Journal of Clinical Pediatric Dentistry (2019): 10.5005/jp-journals-10005-1645
\end{abstract}

\section{INTRODUCTION}

"How" and "why" diseases show a familial distribution has been the goal of many genetic epidemiologists. A better understanding of the relative effects of genes and environment on the dentofacial parameters should improve our knowledge on the etiology of dental problems and also on the possibilities and limitations of the dental treatment and treatment planning. As the standard of community dental health rises, clinical genetics becomes of increasing relevance. An important advantage of clinical dental genetics is the early identification and prophylactic therapy of those "genetically at risk." If the practitioner is aware of the mode of genetic transmission, then members of the family can often be sought and examined early in life and the groundwork may be laid for subsequent dental management.

There have been three major advancements in the use of twins over the past four decades. First, the refinements in the technique of analysis, mainly analyzing twin data to avoid false conclusions, so often made in the past as proposed by Christian and colleagues. ${ }^{1}$ Second, Boklage found a major difference in the development, growth, and behavior in twin families compared with non-twin families. ${ }^{2}$ The third innovation concerns the development of the twin half-sib method. Because of the limited number of offspring from the same parents, the efficiency of the half-sib method could be increased by the large number of monozygotic twin families, as monozygous twins are biologically identical. Genetically, the
1,2,5,6 Department of Pedodontics and Preventive Dentistry, Government Dental College, Thiruvananthapuram, Kerala, India

${ }^{3}$ Department of Prosthodontics and Implantology, Amrita School of Dentistry, Amrita Vishwa Vidyapeetham, Kochi, Kerala, India

${ }^{4}$ Department of Orthodontics, PMNM Dental College and Hospital, Bagalkot, Karnataka, India

Corresponding Author: Jaini Lalithamma, Department of Prosthodontics and Implantology, Amrita School of Dentistry, Amrita Vishwa Vidyapeetham, Kochi, Kerala, India, Phone: +919447461776, e-mail: drjainij@gmail.com

How to cite this article: Zarina R, Kuriakose $S$, et al. Evaluation of Interpair Differences in Caries among South Indian Monozygotic Twins: A Cross-sectional Study. Int J Clin Pediatr Dent 2019;12(4):318-324.

Source of support: Nil

Conflict of interest: None

children of these monozygous twins are a unique class of halfsibs. Several studies have used this powerful method for genetic epidemiological studies. ${ }^{3,4}$

Twins, as first suggested by Galton, form a unique tool to evaluate the interactions between "nature" and "nurture." The interaction between several genes and environmental factors contributes to an individual's susceptibility to a particular 
disorder. ${ }^{5}$ Levin 1985 noted that a common disorder such as dental caries occurring in childhood affect $68 \%$ of five-year-olds, and a majority of 15-year-old children in industrialized countries. ${ }^{6}$ The host factor of the famed Keyes triad presents us with an unexplored avenue for research, that is the genetic aspects of dental caries. ${ }^{7}$ Previous studies involving twins have suggested a genetic influence associated with caries experience. ${ }^{8-17}$ The mechanism whereby this genetic influence exerts its effects has never been isolated because many human trials are polygenic or susceptible to environmental modifications and are difficult to be studied conventionally. Monozygous twins are identical in genetic makeup and sex. The twin method allows an approach to the study of such trials.

It has been accepted that the form of the teeth and jaws are matters of inheritance, hence an assessment of the differences in the various dental attributes of the twins help determine in an exact manner the character and the extent of the influence exercised by the environment, as a complement of the genetic constitution. Dobzhansky wrote that every person is indeed biologically and genetically different from every other, even in the case of identical twins, and there is evidence that differences increase rather than decrease, as children go from childhood into adolescence, and eventually to old age. ${ }^{18} \mathrm{~A}$ review of the related literature showed that several studies were conducted on twins. But a closer analysis of the studies reviewed that a very limited knowledge is available regarding the relationship between birth weight and birth order and the different dental attributes in twins. Hence, a modest attempt was made to fill this gap by taking up a study of the present type.

\section{Materials and Methods}

The study was designed as a cross-sectional census type of study owing to limitations in getting an adequate sample size. Approval for the study was obtained from the Ministry of Health, Government of Kerala. Permission was granted to visit the schools and dental colleges to collect the required data and to perform the clinical examination of children. The research protocol and informed consent form were reviewed and approved by the Institutional Ethical Committee, Government Dental College, Thiruvananthapuram. The steps, procedures, possible discomforts and risks, as well as advantages were explained in detail to the parents. Their informed consent was obtained before the procedure. A pilot study was conducted prior to the actual study, involving 10 pairs of monozygotic twins.

\section{Sample}

The sample consisted of 20 pairs of monozygotic children (aged 6-12 years) from the city of Thiruvananthapuram, Kerala, India. The criteria for case selection in the study were based on determination of zygosity. Marked similarities in facial features, hair whorls, and the color of the eyes were assessed. Heritability of dental characteristics was measured by applying the proposed methods for the statistical analysis of twins, and carefully selecting the twin samples to avoid bias resulting from the manipulation of the phenotype (exfoliated teeth). Also twins who had already undergone and those who were undergoing orthodontic therapy were excluded from the study. Lastly, the most important selection criteria were including only reared-together models. To avoid operator bias, all the samples were assessed by the same operator.

\section{Questionnaire}

The parents of the children recruited for the study were asked to complete the questionnaire (Fig. 1) and report along with their children.

In those cases where a doubt existed regarding the birth weight of child, the medical cum dental examination method was used. The sociodemographic variables such as age, sex, socioeconomic status, birth order; nutritional variables such as birth weight, food habits and preferences, frequency, quantity and type of food; oral hygiene practices such as time of starting of brushing and frequency of brushing and duration for which parents helped for brushing and time of self-starting of brushing practices; and also liking, availability, frequency, and quantity of consumption of sweets were evaluated.

\section{Data Collection}

The examination procedures and diagnostic criteria were those recommended in the World Health Organization Oral Health Survey Basic Methods. The dental examinations were carried out starting from the maxillary right quadrant to the mandibular right quadrant in a regular clockwise manner, using visual and, to some extent, tactile aids. The tip of the explorer was gently used to check for loss of surface smoothness, to determine the base of the cavity, and to assess the plaque and calculus deposition. The DMFT, dmft, DMFs, and dmfs scores were measured to find out the association of the prevalence of dental caries in monozygotic twins and various variables such as socioeconomic status, gender, infant feeding practices, oral habits, brushing habits, and liking for sweets. An inter-pair comparison of the DMFT, dmft, DMFS and dmfs scores in monozygous were carried out. For further comparison, the monozygous twins were grouped based on the birth order and relative to their birth weight into HBWT (high birth weight twin) and LBWT (low birth weight twin).

\section{Statistical Analysis}

The data were transferred into a master sheet and statistical tables were constructed. All calculations were done using the Statistical Package for Social Sciences-SPSS 15 for windows (SPSS Inc., Chicago, IL, USA), and the associations and variations were evaluated. The statistical constants such as mean, standard deviation, and percentage were computed for comparing different variables. The statistical hypotheses formulated were tested by using Chi-square tests to see the association between variables, and the equality of mean scores were tested by using student's " $t$ " tests.

\section{Results}

Sample sizes of 40 monozygotic twins (20 pairs) were selected for this study. Among them twenty were males and twenty were females [total number of samples $=40$, females $=20(50 \%)$ and males $=20(50 \%)]$. Among the total samples $61.5 \%$ exhibited caries. It was noted that among the male twins, $90 \%$ had dental caries and among the female twins $45 \%$ had only caries. Thus, almost two-fold increases in the prevalence of dental caries was observed among male twins in the present study. The observed difference turned out to be highly statistically significant $\left(\chi^{2}=\right.$ $12.1 ; \mathrm{df}=1 ; p<0.01$ ). Therefore, it was inferred that the risk of dental caries was rather high among males compared to female twins (Fig. 2).

When the gender and relative weight of the first born of monozygotic twins were examined, it was seen that 13 of the male 


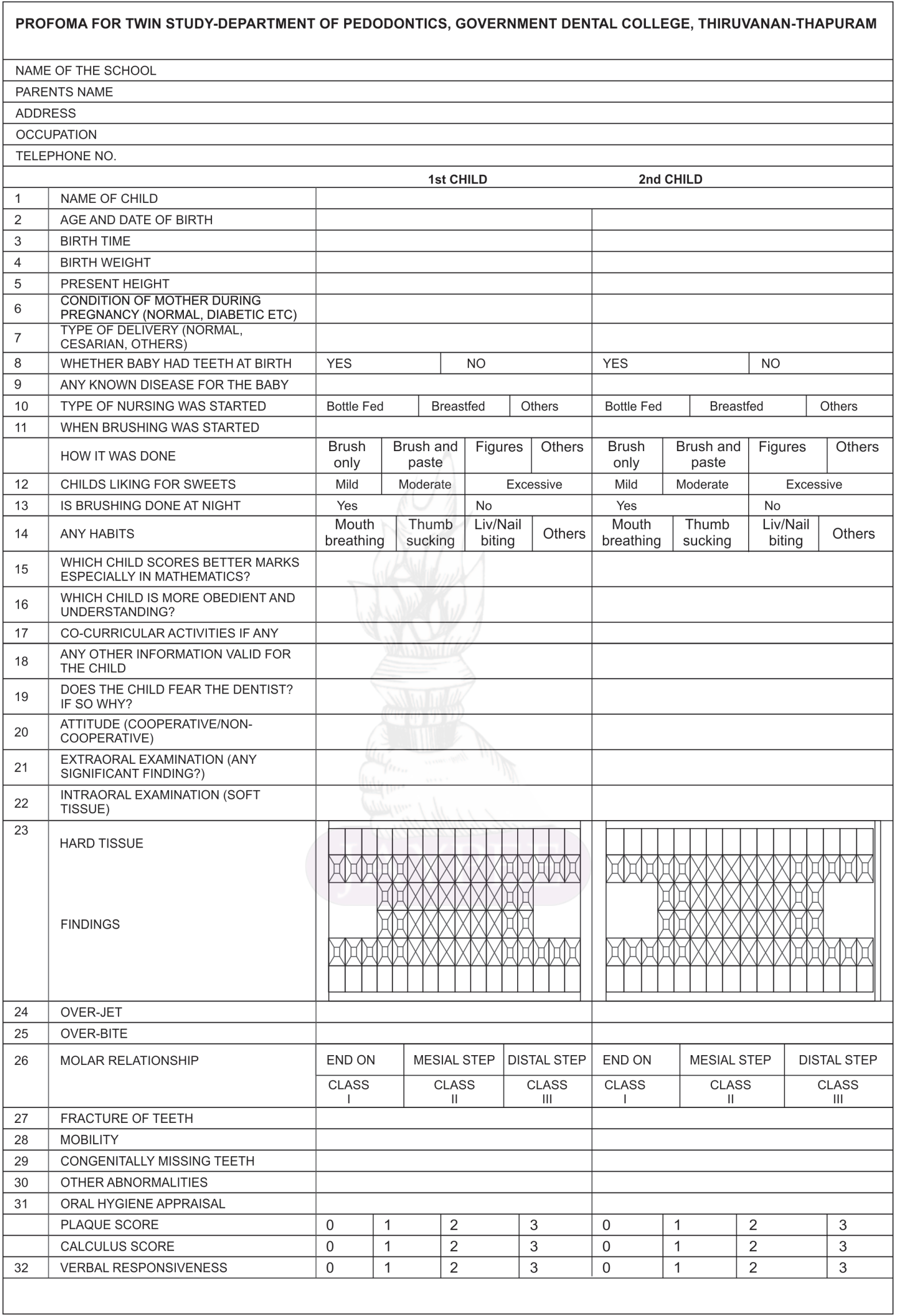

Fig. 1: Questionnaire 


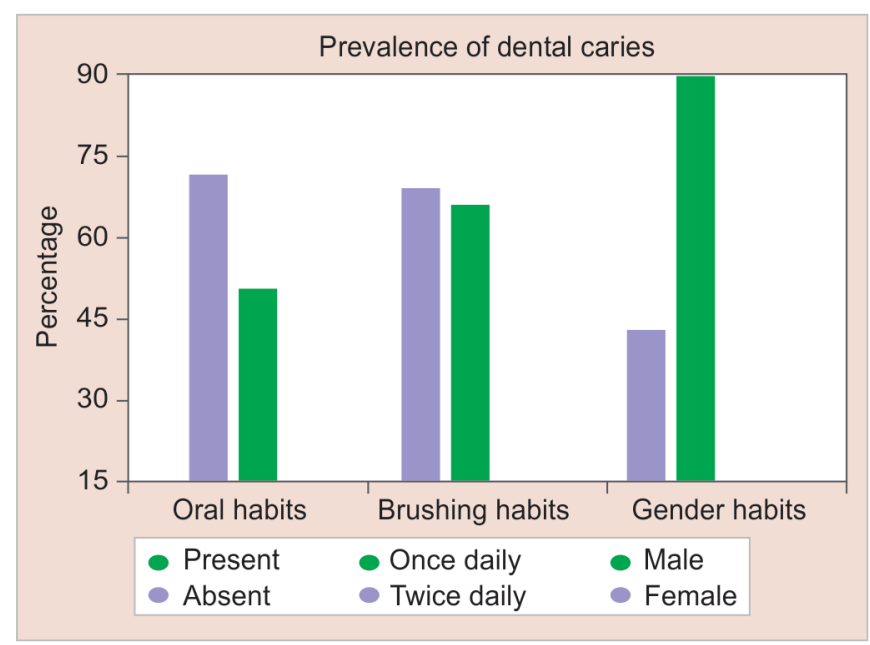

Fig. 2: Relationship between prevalence of dental caries (\%) and oral habits, brushing habits and gender

Table 1: Sex distribution according to the order of birth and relative weight in monozygous twins

\begin{tabular}{llcc}
\hline Gender & High weight twins & Low weight twins & Total \\
\hline Male & 13 & 7 & 20 \\
Female & 11 & 9 & 20 \\
Total & 24 & 16 & 40 \\
Percentage & 60 & 40 & 100 \\
\hline
\end{tabular}

and 11 of the female first-born twins had a relatively higher birth weight than their co twins (Table 1).

It was noticed that 60 percentages of the first born had a relatively higher birth weight than the second born of the twins. While comparing the prevalence of dental caries in these 2 groups, the lower weight twins showed an increased chance of dental caries (72.22\%), compared to higher weight twins (68.18\%) (Fig. 3), where the difference was not established statistically $\left(x^{2}=0.78\right.$; $\mathrm{df}=1$; $t=1.003 ; p>0.05)$. It was interesting to note that the second of the twins had an increased risk of dental caries (70\%) compared to the first of the twins (60\%) (Fig. 3) $\left(x^{2}=1.11 ; \mathrm{df}=1 ; p>0.05\right)$.

It was seen that $75 \%$ of the children did not have any habits such as tongue thrusting, thumb sucking, and lip biting during childhood and the remaining $25 \%$ had such habits. The prevalence of dental caries among children without habits was high (73.3\%) when compared to the other group (50\%) (Fig. 2). The Chi-square test for association showed the difference as significant at $5 \%$ level ( $\left.x^{2}=5.34 ; \mathrm{df}=1 ; p<0.05\right)$. Evaluation of the association between dental caries and the socioeconomic status revealed that, the risk was rather high for the high socioeconomic group (71\%) compared to the other two groups under study, namely middle and low socioeconomic status, which had a caries prevalence of $68 \%$ and $61 \%$, respectively. However, the statistical test revealed no significant association between the socioeconomic status and the prevalence of caries (Fig. 4$)\left(x^{2}=0.64 ; \mathrm{df}=2 ; p>0.05\right)$.

In the present study, only $25 \%$ had exclusive breastfeeding and the majority had a combination of breastfeeding and bottle feeding (55\%). The remaining $20 \%$ were solely dependent on bottlefeed only. Numerically, those who were exclusively breastfed had an increased risk (70\%) of dental caries, while the bottle feed had $62.5 \%$ and both together had $63.6 \%$. Still the association between dental caries and

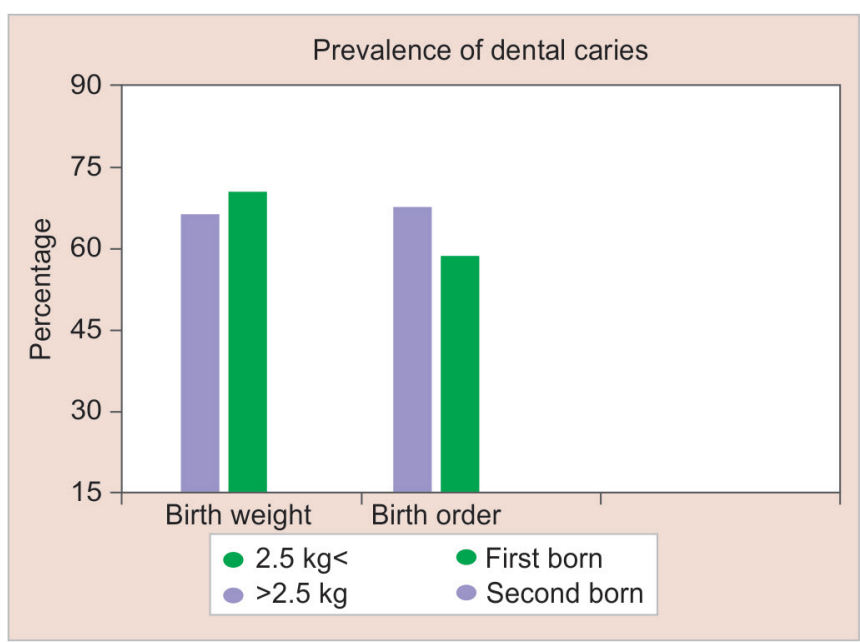

Fig. 3: Relationship between prevalence of dental caries (\%) and birth weight and birth order

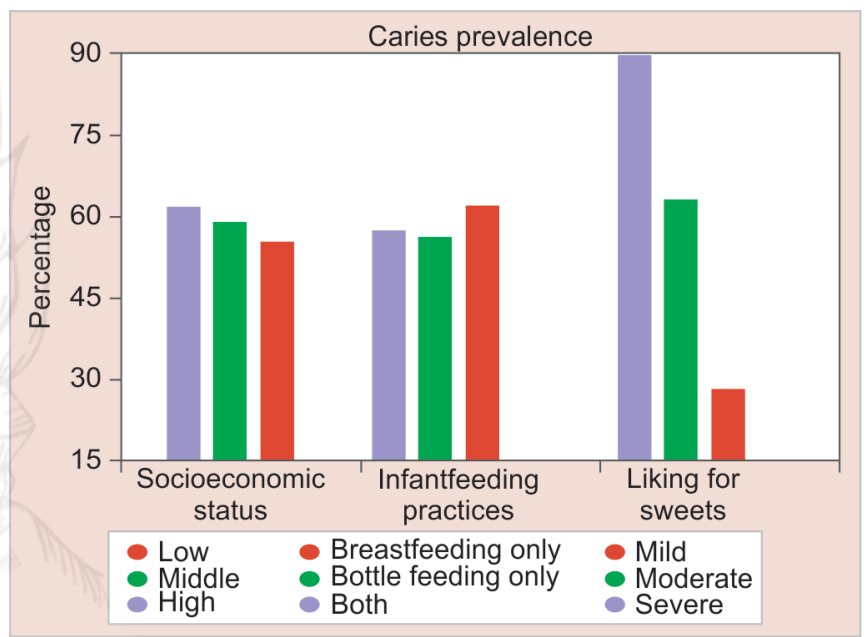

Fig. 4: Relation between caries prevalence (\%) and socioeconomic status, infant feeding practice and liking for sweets

breastfeeding status showed no statistically significant association $\left(x^{2}=2.04 ; \mathrm{df}=2 ; p>0.05\right)$ (Fig. 4). Of the total dental caries, $70 \%$ were among children who brushed twice or more, who started the brushing as early as the onset of eruption of first tooth, whereas it was $66.67 \%$ among children who brushed only once, who started their brushing practices at a later stage (Fig. 2). In fact, the difference was only negligible and the statistical test also substantiated this $\left(x^{2}=0.63 ; \mathrm{df}=1 ; p>0.05\right)$. It was found that among the twins, majority of them learned the brushing and started to practice themselves between the ages of 3-4. A very strong association was noted between excessive sweet liking and the prevalence of dental caries because $100 \%$ of the children who had excessive sweet consumption had dental caries. The minimum percentage of dental caries was reported among those who had only a mild liking for sweets (33.3\%) followed by $73.07 \%$ in moderate group (Fig. 4). The statistical test for association agreed with the numerical findings $\left(x^{2}=16.24 ; \mathrm{df}=2 ; p<0.001\right)$ (Fig. 4). An inter-pair comparison of the DMFT, dmft, DMFS, and dmfs scores in monozygous twins were carried out. Mean \pm standard deviation and $t$ values of dental scores and dental surface scores for high-weight and low-weight monozygous twins were calculated (Tables 2 and 3 ). 
Table 2: Mean \pm standard deviation and $t$ values of dental scores for low-weight and high-weight monozygous twins

\begin{tabular}{|c|c|c|c|c|}
\hline \multirow[b]{2}{*}{ Dental scores } & \multicolumn{2}{|c|}{ Mean $\pm S D$ of score } & \multirow[b]{2}{*}{ " $t$ " value } & \multirow[b]{2}{*}{$p$ value } \\
\hline & Low-weight & High-weight & & \\
\hline DT & $3.48 \pm 2.94$ & $3.96 \pm 3.70$ & 0.29 & $>0.05$ \\
\hline MT & $0.15 \pm 0.43$ & $0.14 \pm 0.47$ & 0.10 & $>0.05$ \\
\hline FT & $0.03 \pm 0.16$ & $0.14 \pm 0.47$ & 0.22 & $>0.05$ \\
\hline DMFT & $3.65 \pm 3.09$ & $3.85 \pm 3.81$ & 0.25 & $>0.05$ \\
\hline $\mathrm{dmft}$ & $2.80 \pm 2.95$ & $2.90 \pm 3.74$ & 0.13 & $>0.05$ \\
\hline
\end{tabular}

Table 3: Mean \pm standard deviation and $t$ values of dental surface scores for low-weight and high-weight monozygous twins

\begin{tabular}{|c|c|c|c|c|}
\hline \multirow[b]{2}{*}{ Dental scores } & \multicolumn{2}{|c|}{ Mean $\pm S D$ of score } & \multirow[b]{2}{*}{ " $t$ "value } & \multirow[b]{2}{*}{$p$ value } \\
\hline & Low-weight & High-weight & & \\
\hline DS & $6.67 \pm 7.27$ & $7.73 \pm 9.98$ & 0.54 & $>0.05$ \\
\hline MS & $0.75 \pm 2.13$ & $0.75 \pm 2.42$ & 0.00 & - \\
\hline FS & $0.08 \pm 0.47$ & $0.13 \pm 0.46$ & 0.22 & $>0.05$ \\
\hline DMFS & $7.53 \pm 8.47$ & $8.60 \pm 10.68$ & 0.51 & $>0.05$ \\
\hline dmfs & $5.90 \pm 7.20$ & $6.63 \pm 10.18$ & 0.37 & $>0.05$ \\
\hline
\end{tabular}

It was noticed that the dmft scores were identical in monozygous twins irrespective of their birth weight. A further attempt to compare the DMFS, dmfs scores showed a numerical rise in the score of the low birth weight twins but did not show any statistical significance.

\section{Discussion}

The present study showed that among the total samples $61.5 \%$ exhibited caries. Of the male children, $90 \%$ had dental caries while only $45 \%$ of the female twins showed caries. When caries incidence of twins was compared with respect to the birth order, it was noted that the second of the twins had an increased risk of dental caries (70\%) compared to the first-born twin. It was noticed that sixty percentages of the first born had a relatively higher birth weight than the second born of the twins. While comparing the prevalence of dental caries in these 2 groups, the lower birth weight twins showed an increased chance of dental caries (72.22\%), compared to higher birth weight twins (68.18\%). The etiology of dental caries has been attributed to the interaction between four essential factors: a cariogenic diet, a caries-producing bacterial flora, a susceptible tooth, and time. ${ }^{7}$ The first three factors encompass a variety of biologically complicated entities such as plaque, saliva, tooth matrix formation, and crystallization, making it easy to visualize that the development of these complex elements must involve a great number of genes. It is revealed from many dietary studies, notably the Vipeholm study by Gustafsson et al., that personal variations exist in susceptibility to dental caries, even under identically controlled circumstances. ${ }^{19}$

Mckeag and Scott proposed that dissimilarity in identical twins was usually thought to be the result of environmental factors. ${ }^{20}$ But before considering these, other possible causes should be eliminated. Gates has suggested that there could be a maternally monozygomatic and paternally dizygomatic twins, where the ovum has divided before fertilization and each divided part is fertilized by a separate sperm. Also, it was not certain as to when the division of the zygote took place, he reasoned that the later the division occurred, the greater was the probability of differences between the members of the twin pair. Thus, conjoint twins usually showed marked differences in height, weight, features, and intelligence. ${ }^{21}$ However, Newmann argued that in cases when twin fetuses were sharing the same placenta, a difference in the blood supply due to an unequal blood exchange may cause a difference in size that was more prominent in identical fetuses. He laid emphasis on the asymmetry mechanism and the time of zygotic division. He stated that, as there was abundant evidence that monozygotic twins were frequently derived through the bilateral fission of prospective right and left sides of the embryo, there would be difference in character expression in the two portions of some monozygous pairs as occurs on the two sides of single individuals. He found that about $25 \%$ of cases identical twins showed the phenomenon of mirror imaging, in which one half of one twin is more like the opposite half of the other, than its own opposite half. ${ }^{22}$

The study revealed that there was almost a twofold increase in the incidence of dental caries among male twins. The significance noted may be attributed to the special care taken in rearing males. The study depicted that $75 \%$ of the children did not have any oral habits and only $25 \%$ had deleterious habits. The prevalence of dental caries was seen to be very high (73.3\%) among children without oral habits. It may thus be presumed that the reduction in caries is due to the increased oral clearance and the action of the oral musculature on the teeth at the time of practicing the habit. Though Wilmot has studied the oral habits and their association to malocclusion, the relationship of habits to caries has not been dealt with. ${ }^{23}$ The relationship between caries and socioeconomic status was studied by Turin et al. and it was seen that those children belonging to a higher socioeconomic status had higher caries prevalence than low and middle socioeconomic groups, which agrees with the present study. ${ }^{24}$

In the present study, the infant feeding practices were estimated to be $25 \%$ in those who had exclusive breastfeeding, $20 \%$ in the bottlefed, and $55 \%$ having a combination of breast and bottle feeding. Numerically, the breastfed children had an increased risk of dental caries (70\%) compared to the other groups. Although the breast milk is known to contain both antibodies and immunologically competent cells and is thought to provide passive immunity for the body, a growing tendency on the part of young mothers to use nursing as a pacifier can be more or less responsible for the increase in prevalence of caries. This is true whether nursing is from the breast or from the bottle. In a study by Kuriakose and Joseph, it was seen that lack of breastfeeding and prolonged breastfeeding beyond 12 months are significant factors for the caries prevalence. ${ }^{25}$ The study also showed that $25 \%$ of children brushed their teeth twice or more and only $75 \%$ once daily. The differences noticed were negligible. Thus, it leads to the conclusion that the frequency of brushing might not have much influence over caries, which is contrary to the popular expectations. A very strong association was noted between excessive liking for sweets and the prevalence of caries because $100 \%$ of the children who had excessive liking and hence possibility of excessive sweet consumption had dental caries. Only a minimum percentage of caries was reported among those who had only a mild liking (22.5\%) and among those who had a moderate liking for sweets (26\%). Pados et al. conducted a study on the eating habits of 111 monozygous and 67 like sexed dizygous twins with respect to cariogenic foods. A biometrical analysis showed that the eating habits of the monozygous pairs were more similar than that dizygous twin pairs. The results were found to be statistically significant. On the basis of these findings, he emphasized the fact that genetic factors play a role in the development of the food habits. ${ }^{26}$ 
Chung and Chiang in their study on 280 pairs of same-sex twins compared the variability of dental caries, missing and filled surfaces (DMFS), the concordance, and higher correlation in monozygous and dizygous twins. The heritability index was $8.7 \%$ according to this study. Hence, it was suggested that environmental influence was dominant in caries initiation, whereas heredity was of little influence. ${ }^{27}$ Gao studied the variability of dental caries, missing and filled surfaces in monozygous and dizygous twins and found that there was less variance, higher concordance, and higher correlation in monozygous twins, though the results were not statistically significant. The author concluded that environmental influence was dominant in caries initiation, whereas heredity was of little influence. ${ }^{28-31}$ Lundstrom while summarizing a number of investigations of these types concluded that about $40 \%$ of the dental and facial variations can be attributed to hereditary factors. ${ }^{32}$

\section{CONCLUSION}

The cross-sectional study done on 40 monozygotic twins in the age group of 6-12 years, to evaluate the determinant differences in the dental attributes, revealed the following observations.

- An inter-pair comparison of monozygous twins showed that the twins with a relatively lower birth weight had a numerically increased prevalence of dental caries, which could not be proved statistically. The mean dental scores of DMFT, dmft, DMFS, dmfs were almost the same for low and high birth weight monozygous twins.

- Twofold increases in caries prevalence was noted among male than female twins. The observed difference turned out to be statistically significant.

- The second-born twin had a numerical increase of caries prevalence compared to the first born, though the association was not established statistically.

- No significant association was found between caries and infant feeding practices and also between socioeconomic status and dental caries. A significant positive relationship was established between caries in twins and the absence of oral habits. It was noticed that the brushing frequency was not related to the severity of caries. A highly significant association between the liking for sweets and dental caries was seen.

\section{What this Paper Adds}

Very little is known about the relationship between birth weight and birth order and the different dental attributes in twins. Hence, a modest attempt was made to fill this gap by taking up a study of the present type. The study provides an assessment of the differences in the prevalence of caries and their relationship to other variables among the monozygotic twins; this will help determine exactly the character and the extent of the influence of the environment as the complement of the genetic constitution.

\section{Clinical Significance of Study}

If the mode of genetic transmission is revealed, this will enable early identification of those who are at risk, thereby making subsequent dental management easy. Hence this is an increasingly important area of dental research. Twin studies are a strong tool that explores these hidden aspects.

\section{ACKnOWLedgments}

Authors acknowledge Dr Pradeep Samuel and Jyothy for their support while editing the article.

\section{References}

1. Christian JC, Kang KW, et al. Choice of an estimate of genetic variance from twin data. Am J Hum Genet 1974;26:154-161.

2. Boklage $\mathrm{CE}$. On the distribution of non-right handedness among twins and their families. Acta Genet Med Gemellol 1981;30:167-187. DOI: $10.1017 /$ S0001566000007649.

3. Christian JC. Use of twins to study environmental effects. Environ Health Perspect 1981;42:103-106. DOI: 10.1289/ehp.8142103.

4. Vlietnick RF. Determination of the zygosity of twins. Leuven, Belgium: Catholic University of Leuven; 1986.

5. Galton F. The history of twins as criterion of the relative powers of nature and nurture. J Anthrop Inst 1985;5:391-406.

6. Levin RS. The scientific basis of dental health education. Brit Dent J 1985;159:223-226. DOI: 10.1038/sj.bdj.4805576.

7. Keyes PH. Present and future measures for caries control. J Am Dent Assoc 1969;79:1935-2404. DOI: 10.14219/jada.archive.1969.0037.

8. Boklage CE. On the distribution of non-right handedness among twins and their families. Acta Genet Med Gemellol 1981;30:167-187. DOI: $10.1017 /$ S0001566000007649.

9. Boraas JC, Messer LB, et al. A genetic contribution to dental caries, occlusion and morphology as demonstrated by twins reared apart. J Dent Res 1988;67:1150-1155. DOI: 10.1177/00220345880670090201.

10. Caldwell RC, Finn SB. Comparison of the caries experience between identical and fraternal twins and unrelated children. J Dent Res 1960;39:693-694.

11. Bachrack FH, Young M. A comparison of the degree of resemblance in dental characters shown in pairs of twins of identical and fraternal types. Br Dent J 1927;48:1293-1304.

12. Nakata $M$, Yes $P$, et al. Genetic determinants of cranio-facial morphology: A Twin Study. Am Hum Genet 1974;37:341-443. DOI: 10.1111/j.1469-1809.1974.tb01848.x.

13. Conry JP, Messer LB, et al. Dental caries and treatment characteristics in human twins reared apart. Arch Oral Biol 1993;38:937-943. DOI: 10.1016/0003-9969(93)90106-V.

14. Potter RH, Nance WE. A Twin study of dental dimensions. II. Discordance, asymmetry and mirror imagery. Am J Phys Anthropol 1976;44:391-396. DOI: 10.1002/ajpa.1330440303.

15. Corrucini RS, Potter RHY. Genetic analysis of Occlusal variation in twins. Am J Orthod 1980;78:140-154. DOI: 10.1016/00029416(80)90056-1.

16. Biggerstaff RH. Morphological variations for the permanent first molars in human monozygotic and dizygotic twins. Arch oral Biol 1970;15:721-730. DOI: 10.1016/0003-9969(70)90036-1.

17. Staley RN, Green LJ. Bilateral asymmetry in tooth cusp occurrence in human monozygotic twins, dizygotic twins and non twins. J Dent Res 1971;50:893. DOI: 10.1177/00220345710500013601.

18. Dobzhansky T. Differences are not defects. Psychology Today 1973;7:96-101.

19. Gustafsson BE, Quensel CE, et al. The Vipeholm dental caries study: The effect of different levels of carbohydrate intake on caries activity in 463 induviduals observed for five years. Acta Odont Scand 1954;11:232-364. DOI: 10.3109/00016355308993925.

20. McKeag HTA, Scott JH, et al. The Aetiology of Irregularity and Malocclusions of the teeth. 2nd ed., Dental Board of the United Kingdom; 1929. pp. 321-326.

21. Gates RR. Human Genetics. New York: Macmillan; 1946.

22. Newmann HH, Frank N, et al. Twins: A study of Heredity and Environment. Chicago: University of Chicago Press; 1937.

23. Wilmot DR. Thumb sucking habit and associated dental differences in one of MZ twins. Br J Orthod 1984;11:195-199.

24. Truin GJ, König KG, et al. Time trends in caries experience of 6-12 year old children of different SES in Hague. Caries Res 1998;32:1-4. 
25. Kuriakose $\mathrm{S}$, Joseph E. Caries prevalence and its relation to socioeconomic status and oral hygiene in 600 pre-school children of Kerala-India. J Indian Soc Pedo Prev Dent 1999;17:97-100.

26. Pados R, Metneki J, et al. A comparative study of the consumption of cariogenic food in M.Z and same sex Dizygotic twins. Orv Hetil 1989;130:509.

27. Chung $\mathrm{H}$, Chiang HTC. Dental caries in $\mathbf{2 8 0}$ pairs of same-sex twins. School Stomatol 1990;25:18-20.

28. Gao XJ. Dental caries in 280 pairs of same sex twins. Zhonghua Kou Qiang Yi Xue Za Zhi 1990;25:18-20.
29. Townsend G, Hughes $T$, et al. Genetic and environmental influences on human dental variation: a critical evaluation of studies involving twins. Arch Oral Biol 2009;54:45-51.

30. Werneck Rl, Mira MT, et al. A critical review: an overview of genetic influence on dental caries. Oral Dis 2010;16:613-623.

31. Moreira MJS, Schwertner C, et al. Dental caries and associated factors in twins with Down syndrome: a case report. Spec Care Dentist 2016; $1-4$.

32. Lundstrom A. Nature vs nurture in dentofacial variation. Eur J Orthod 1984;15:153-162. 